\title{
La organización de eventos en el contexto de las Comunicaciones Integradas de Marketing (IMC): el valor de la experiencia
}

\author{
María GALMÉS CEREZO ${ }^{1}$ \\ Juan Salvador VICTORIA MAS ${ }^{2}$
}

Universidad de Málaga

\begin{abstract}
RESUMEN:
En la última década se ha producido un importante desarrollo de la organización de eventos dentro de la estrategia de comunicación de marketing de las empresas. Esta tendencia está relacionada, por supuesto, con el hecho de que han demostrado ser una herramienta eficaz, pero no eficaz de cualquier manera, sino al modo en que el marketing (el público) actual parece que lo está demandando: a través de experiencias que llevan a la emoción, a la relación, al sentimiento, al pensamiento y a la actuación con las marcas, las empresas o sus productos. Las Comunicaciones Integradas de Marketing (IMC) tienen por tanto en la organización de eventos una herramienta capaz de aportar valor a las marcas a partir de la relación experiencial con ellas.

El objetivo de este estudio es analizar la organización de eventos en el contexto de las IMC: detectar los principales aspectos que ofrezcan las claves de cómo los organizadores de eventos pueden generar experiencias que les permitan alcanzar unos objetivos concretos, al tiempo que evaluar sus resultados.

En este artículo se examinan las aportaciones de expertos en organización de eventos, y de teóricos de las IMC; así como las propuestas académicas y profesionales relativas a la experiencia de marca y al marketing experiencial. A partir de las relaciones entre ellas se ha realizado una propuesta teórica sobre el papel que pueden tener los eventos en la estrategia de comunicación de marketing, y su capacidad para generar experiencias con las marcas.
\end{abstract}

PALABRAS CLAVE: Organización de eventos; Comunicaciones de Marketing Integradas (IMC); Experiencia de Marca; Eventos de Comunicación de Marketing (ECM).

TITLE: The organization of events in the Integrated Marketing Communications (IMC) context: the value of experience

\begin{abstract}
:
In the last decade has been significant development of the organization of events within the marketing communication strategy of companies. This trend is related, of course, that proved to be an effective tool, but not effective in any way, but the way that the marketing (the public) now seems that it is demanding, through experiences lead to the emotion, relationship, feeling, thought and action with the brands, companies or products.

Integrated Marketing Communications (IMC) are both in the organization of events with a tool capable of adding value to brands from the experiential relationship with them. The aim of this study is to analyze the organization of events in the context of the IMC: identifying key aspects which offer clues as to how event organizers can generate experiences that enable them to achieve specific objectives, while evaluating the results.

This article examines the contributions of experts in event management, and theorists of the IMC, as well as academic and professional proposals relating to the brand experience and experiential marketing. From the rela-

\footnotetext{
Doctora en Publicidad y RR.PP. Universidad de Málaga

2 Profesor Titular de Comunicación Audiovisual y Publicidad en la Universidad de Málaga. Profesor
} visitante en la Northwestern University (Chicago, IL).
\end{abstract}


tionship between them has been made a theoretical proposal about the role they can play events on the marketing communication strategy, and ability to create brand experiences.

KEY WORDS: Events management, Integrated Marketing Communications (IMC); Brand Experience; Marketing Communication Events (ECM).

\section{Introducción}

Hace unos años el término «comunicaciones de marketing» se utilizaba para nombrar a una serie de herramientas que se planificaban de manera independiente $\mathrm{y}$, a veces, de forma inconexa. ${ }^{3}$

En la actualidad, una de las preocupaciones fundamentales de las empresas tiene que ver con la integración estratégica de todas las herramientas de comunicación para transmitir a la audiencia mensajes coherentes. Es decir, los planteamientos han evolucionado hacia las «Comunicaciones Integradas de Marketing (IMC)», que parten de un nuevo modo de concebir y llevar a cabo el mix de comunicación.

Victoria ${ }^{4}$ expone que el origen del término se puede hallar en la Northwestern University. Fue allí donde Caywood ${ }^{5}$ y otros expertos utilizaron el término por primera vez. Desde entonces son muchos los trabajos académicos anglosajones dedicados a analizar las IMC en profundidad ${ }^{6}$.

Las IMC tratan de conseguir una coordinación efectiva entre todas las herramientas de comunicación (venta personal, publicidad, relaciones públicas, marketing directo, organización de eventos, marketing online, etc.), y el resto de las actividades de la empresa que puedan influir en la imagen de los públicos de la marca.

Los públicos integran todos estos mensajes sobre la marca, y no distinguen de donde proceden. Por ello la empresa necesita integrar todas las herramientas para conseguir transmitir un mensaje consistente sobre la marca, producto u organización.

En la actualidad aunque la publicidad sigue siendo imprescindible para muchas empresas, se están contemplando otras herramientas en las estrategias de comunicación. El objetivo es tratar de aprovechar las ventajas que pueden aportar cada una de estas herramientas a la estrategia integrada. Esto supone un gran esfuerzo para coordinarlas de forma adecuada. Para ello, según el protocolo descrito por las propias IMC, sería necesario conocer los resultados de las comunicaciones realizadas, evaluar su eficacia a través de criterios objetivos, y-finalmente- conocer su impacto sobre el mensaje glo-

\footnotetext{
3 Kitchen, J. y De Pelsmacker, P (2004): Integrated Marketing Communications. A primer. New York, Routledge.

4 Victoria, J.S. et al (2005): Reestructuras del Sistema publicitario. Barcelona, Ariel, 44.

5 CAywood, C.L. (1997 ): The Handbook of Strategic Public Relations \& Integrated Communications. McGraw-Hill Professional.

6 Belch y Belch. 2009; Clow y BaAck, 2005; Holm, 2006; Kitchen y De Pelsmacker, 2004; Pickton y Broderick, 2005; SchultZ y Schultz, 2004; Shimp, 2008.
} 
bal. Para Rodríguez Ardura ${ }^{7}$ este último representaría el reto más importante de la aplicación de la Estrategia de las Comunicaciones de Marketing Integradas.

\section{El concepto Eventos de Comunicación de Marketing}

Para entender esta perspectiva, que considera a los eventos como herramientas que se integran en la estrategia de comunicación de marketing, es necesario acotar y definir un nuevo concepto: «Eventos de Comunicación de Marketing» (ECM). Este nuevo concepto es el resultado de una evolución lógica, que parte de los «eventos de empresa», pasa por los «eventos corporativos», y llega hasta los «eventos de comunicación de marketing». Su utilización se hace necesaria en el momento en que la organización de eventos empieza a ser considerada como una herramienta capaz de aportar valor dentro de la estrategia de comunicación de marketing de las empresas.

En este momento las organizaciones necesitan integrar todas las herramientas para conseguir transmitir un mensaje consistente y coherente sobre la marca, producto $\mathrm{u}$ organización. Y los eventos son considerados por los profesionales y los académicos, como una herramienta que se integra en esta estrategia ${ }^{8}$. Por otra parte, los profesionales empiezan utilizar los eventos para generar experiencias de marca con sus públicos, lo que les permite conseguir unos objetivos concretos de comunicación de marketing.

Para poder perfilar una definición clara y significativa de los ECM, se ha realizado un análisis documental en profundidad sobre los conceptos «eventos de empresa», «eventos de comunicación» ${ }^{10}$, «eventos de marketing» ${ }^{11},\left\langle\langle\text { marketing de eventos }\rangle^{12}, \mathrm{y}\right.$ «eventos de marketing experiencial» ${ }^{13}$. Se ha sintetizado en la siguiente definición: «Actos presenciales - organizados y planificados dentro de la estrategia de comunicación de marketing de una organización- con el fin de trasladar un mensaje sobre sus productos, marcas o sobre la propia organización, a un público objetivo, para provocar

\footnotetext{
7 Rodríguez ARduA, I. (2007): Estrategias y Técnicas de Comunicación. Una visión Integrada en el Marketing. Editorial OUC, 31

8 Masterman, G. \& Wood, E. (2006): Innovative Marketing Communications: Strategies for the Events Industry. Oxford, Elsevier Buterworf-Heinemann.

9 TORRENTS, R. (2005): Eventos de Empresa. El poder de la comunicación en vivo, Bilbao, Deusto.

10 Acebal NeU, A. (2004): «Eventos: Un instrumento de comunicación al servicio de la empresa». Ediciones Deusto (e- Deusto Dossier) y GuTIÉRREZ ZúMEL, C. (2004): El evento: un plus en comunicación, Bilbao, Ediciones Deusto - Planeta de Agostini Profesional y Formación.

${ }_{11}$ MASTERMAn, G. \& WOOD, E. (2008): «Event Marketing: Measuring an experience». Venice: 7th International Marketing Trends Congress - Jan 17 th $/ 19^{\text {th }}$. Y WOHLFEIL, M. y WHELAN, S. (2005): «Event Marketing: When Brands Become 'Real Lived' Experiences» Irish Academy of Management Conference, Galway-Mayo Institute of Technology.

12 Fenrnstrom, M. y Rich, D. (2009): «Using events to drive an Integrated Marketing Model». Northwestern University, Journal of Integrated Marketing Communications y MOLERO, V. y MORENO, F. (2008): «Marketing de Vanguardia», en Manual de Marketing, Madrid, Editorial ESIC. ESIC.

13 Lenderman, M. (2008): Marketing Experiencial. La Revolución de las Marcas, Madrid, Editorial
} 
una respuesta, o para reforzar, crear o modificar una actitud, a partir de la experiencia del público».

Una vez definido el término, podemos decir que cualquier evento puede ser considerado (con mayores o menores posibilidades) como un ECM. Un evento es un acontecimiento con una audiencia. Al existir una audiencia, un mensaje o experiencia está siendo compartido, transmitido y generado. Por lo tanto, todos los eventos podrían potencialmente comunicar algo. O lo que es lo mismo: todos los eventos pueden ser utilizados por las organizaciones para comunicarse con sus públicos ${ }^{14}$.

No obstante los ECM serían siempre eventos creados expresamente para alcanzar objetivos de comunicación de marketing. En este caso se excluirían los eventos que han ido creados para otros propósitos pero que después son usados para comunicaciones de marketing por terceros, como son los eventos deportivos o culturales que son patrocinados.

Los profesionales del marketing tienen que asumir el riesgo de avanzar en la búsqueda de prácticas innovadoras en sus comunicaciones. Los ECM, tal y como han sido definidos, se encuentran dentro de este paisaje de la necesaria evolución, y están demostrando su utilidad.

\section{El papel de los ECM en la estrategia de Comunicaciones Integradas de Marketing (IMC)}

En los últimos años los eventos han adquirido mayor relevancia en las estrategias de comunicación de marketing de las empresas. Algunas herramientas que se utilizaban tradicionalmente están perdiendo eficacia, y los responsables de comunicación han aumentado los presupuestos destinados a la organización de eventos ${ }^{15}$.

En el estudio de mercado del grupo Eventoplus del 2009 queda patente, que los eventos están jugando un papel cada vez más protagonista en las estrategias de IMC de las empresas españolas. Los responsables de comunicación de las organizaciones consideraron que una media del $22 \%$ de los presupuestos de comunicaciones de marketing se dedica a eventos ${ }^{16}$. Esto supone que los ECM están alcanzando mayor valor y reconocimiento. Y, consecuentemente, la disciplina de la organización de eventos se está expandiendo desde sus orígenes culturales y de celebración, hacia el papel que pueden jugar en las estrategias de marketing de las organizaciones.

14 Masterman, G. \& WoOD, E. (2008): «Event Marketing: Measuring an experience». Venice: 7th International Marketing Trends Congress - Jan 17th $/ 19^{\text {th }}$.

15 Esta afirmación puede resultar excesivamente tópica, y no es la única razón para la evolución de esta herramienta, por lo que ha sido tomada como punto de partida, para ser matizada más adelante.

16 Grupo Eventoplus (2010): «El Estudio del Mercado de Eventos 2010». Disponible en www.eventoplus.com 
La integración de los eventos en la estrategia de comunicación de marketing, y la coordinación con las otras herramientas, es fundamental para asegurar un mensaje consistente $^{17}$.

Algunos autores consideran que los ECM son utilizados para comunicar de una forma mucho más directa que otras herramientas más distantes. Por ello deben buscar la interacción, en vez de una simple invitación a la audiencia a observar o presenciar. Si no se produce esta interacción no tendría sentido realizar el evento y se estaría desaprovechando la ventaja de lo presencial, ya que seguramente el mensaje se podría transmitir a través de otras herramientas de comunicación con un menor coste por impacto. Por tanto, el evento es creado específicamente para alcanzar un objetivo, y esto significa que necesitará un diseño cuidadoso integrado con otras herramientas de comunicación. Desde esta perspectiva, el componente fundamental del evento será la participación de la audiencia y la interacción ${ }^{18}$.

Los ECM han pasado de ser una herramienta que se utilizaba, muy de vez en cuando, para apoyar una campaña publicitaria y reforzar un mensaje de marca, a convertirse en una herramienta fundamental en la estrategia de comunicación integrada ${ }^{19}$.

Este planteamiento quedó confirmado a partir de la investigación encargada por la empresa Jack Morton Worldwide ${ }^{20}$, que dejó de manifiesto las posibilidades de los ECM dentro de la estrategia de comunicación de marketing. A partir de las respuestas de los entrevistados en este estudio se pudo concluir que los ECM aumentan la eficacia de otras herramientas de IMC, que pueden influir sobre la intención de compra, y que son especialmente útiles para generar «rumor» o «boca a boca». Además, los entrevistados manifestaron estar interesados en tener experiencias directas con las marcas, y una de las cosas que más valoraron de los eventos fueron las experiencias interactivas y entretenidas, así como la posibilidad de establecer un diálogo con la marca. También se pudo observar que los jóvenes están especialmente motivados a adoptar las marcas con las que han interactuado en un ECM.

Las conclusiones obtenidas en este estudio cambiaron la forma de entender los eventos que tenían muchos expertos en comunicación, ya que empezaron a desvelar las posibilidades de los ECM para las IMC. Así mismo, han servido para orientar a los responsables de las comunicaciones de marketing en cómo utilizar la organización de eventos para obtener la máxima eficacia, creando experiencias con las marcas.

Algunos expertos consideran que el paradigma de las comunicaciones de marketing está cambiado. Dentro de esta nueva corriente han surgido voces como el manifiesto

17 BERRIDGE, G. (2007): Events Design and Experience, Oxford, Elsevier Buterworf-Heinemann (Events Management Series), 51

18 Ibídem, 8. Pag. 5-10

19 Ibídem, 13. Pag. 145

20 Worldwide Survey on Experiential Marketing: Los resultados se publicaron en el 2004, y se realizó una ampliación en el 2005. (Jack Morton, 2008). 
Cluetrain $^{21}$, o el manifiesto IXMA ${ }^{22}$ que postulan planteamientos como que «los mercados son conversaciones», o que «los consumidores quieren respeto, reconocimiento y relevancia en la comunicación, y han mostrado que el mejor camino para dárselo es a través de experiencias que son personalmente relevantes, memorables, sensoriales, emocionales y significativas». En este nuevo contexto la organización de eventos se propone como una herramienta muy interesante para que las empresas se relacionen con sus públicos a partir de conversaciones, y de experiencias. Y los expertos en marketing experiencial así lo han entendido.

Por otra parte, la integración de las herramientas de comunicación consigue que las ventajas que aporta cada una de las herramientas, compense las limitaciones de las otras herramientas. Los resultados de cada herramienta de comunicación se refuerzan con los resultados de las otras herramientas con las que se integra ${ }^{23}$.

Con la adopción de este enfoque integrador ha cambiado la forma de planificar. Ya no se trata de maximizar la eficacia de las comunicaciones sobre audiencias masivas para un presupuesto dado, ahora se busca entrar en relación con el público objetivo utilizando tipos de comunicaciones más acordes con sus estilos de vida. Se busca alcanzar a un público objetivo segmentado, en el momento ideal, con el mensaje apropiado, generando experiencias positivas.

Por tanto, podemos decir que la capacidad de los eventos para generar experiencias de marca es su valor principal en la estrategia de comunicación de marketing. Los ECM pueden reforzar los resultados de otras herramientas menos experienciales, y a su vez, sus resultados pueden ser mejorados al conseguir ampliar la experiencia a través de otras herramientas de mayor alcance.

\subsection{Los ECM como generadores de experiencias de marca}

Los miembros de IXMA ${ }^{24}$ consideran que los consumidores actuales esperan que las marcas les proporcionen una experiencia, no un mensaje más, esperan campañas que deslumbren sus sentidos y estimulen sus mentes.

El objetivo del marketing experiencial es crear, a partir de estas experiencias, conexiones directas y significativas entre la marca y los clientes. La organización de eventos está demostrando ser una herramienta con una gran capacidad para ofrecer estas experiencias, a un público muy segmentado, de una forma muy directa. $\mathrm{Y}$ de ahí se van a derivar sus principales ventajas respecto a otras herramientas integradas.

21 El manifiesto "The Cluetrain" (www.cluetrain.com), ha sido desarrollado por algunos profesionales de marketing innovadores, que defienden la idea de "conversar" con sus clientes, y buscan formas de general diálogos respetuosos con ellos.

22 IXMA: International Experiential Marketing Asociation. Esta formada por los principales expertos internaciones en marketing experiencial. El "Manifiesto IXMA" incluye varias sentencias que postulan que los públicos buscan relacionarse con las marcas a partir de experiencias.

23 Kitchen, J. y De Pelsmacker, P (2004): «Integrated Marketing Communications. A primer». Journal of Marketing Communications.

24 Ibídim, 22 
Schmitt ${ }^{25}$ ha desarrollado un marco conceptual para generar y gestionar las experiencias de los públicos, que puede ser adaptado a la creación de la experiencia de un ECM. Se centra en dos conceptos fundamentales: los «Módulos Experienciales Estratégicos» (MEEs) y los «Proveedores de Experiencias» (ExPros)

Los «Módulos Experienciales Estratégicos» son los diferentes tipos de experiencias, definidos a partir de unas estructuras y de unos principios. Y los «Proveedores de Experiencias» son las herramientas por medio de las que se crean los MEEs.

Las experiencias son sucesos privados que se producen como respuesta a un estímulo concreto. Los profesionales de marketing pueden aportar estímulos que den como resultado unas experiencias concretas del público objetivo. Deben seleccionar los ExPros adecuados para generar una determinada experiencia de marca. A partir de esta experiencia los públicos van a sentirse más implicados con la marca y más motivados a la acción.

Las experiencias son estructuras muy complicadas. Es cierto que no existen dos experiencias iguales, pero sí se pueden clasificar, en función de sus propiedades, en diferentes tipos de experiencias. Así, los responsables de marketing podrán definir qué tipo de experiencias deben proporcionar para conseguir una determinada respuesta o un cambio de actitud y/o relación con la marca.

Las experiencias pueden diseccionarse en diferentes módulos, cada uno con sus diferentes estructuras y procesos. Se pueden definir cinco tipos de «Módulos Experienciales»: MEE de sensaciones, MEE de sentimientos, MEE de pensamientos, MEE de actuaciones y MEE de relaciones.

Estos «Módulos Experienciales» han sido definidos a partir de un análisis en profundidad de las aportaciones sobre las «Dimensiones de la Experiencia» descritas desde diferentes campos de conocimiento ${ }^{26}$ : desde la filosofía y la antropología de la experiencia, desde la psicología, y desde la gestión empresarial y el marketing. El «MEE de sensaciones» apela a los sentidos generando experiencias sensoriales a través de los cinco sentidos. El «MEE de sentimientos" apela a las emociones mas internas de los clientes, y puede crear experiencias afectivas hacia la marca. El «Módulo de pensamientos» trabaja sobre al intelecto generando experiencias cognitivas que resuelven problemas y que atraen al público creativamente. El «Módulo de actuaciones» puede generar experiencias físicas e interactuaciones con la marca, mostrándoles otras formas de hacer las cosas y enriqueciendo sus vivencias. $\mathrm{Y}$ el «MEE de relaciones» apela a las relaciones sociales, trata de generar relaciones optimas con su yo ideal y con otras personas, y actúa sobre la necesidad de ser percibidos de forma positiva y la necesidad de pertenencia grupal.

25 Sснмітт, B. H. (2006): Experiential marketing : cómo conseguir que los clientes identifiquen en su marca sensaciones, sentimientos... relaciones. Deusto. 80-90.

26 SChmitT , B. H; BRAKuS, J. \& ZARANTONELlo, L. (2008): «Brand Experience: What is It? How do We Measure It? And Does It Affect Loyalty?». Journal of Marketing 
Las estrategias experienciales no se suelen basar en un único tipo de MEE. Se suelen apoyar en «híbridos experienciales» que combinan dos o mas MEEs. O incluso en «experiencias holísticas» que combinan los cinco Módulos Experienciales.

Las decisiones estratégicas de marketing experiencial se deben tomar a partir de una cuidadosa planificación. En primer lugar, habría que decidir qué enfoque se le va a dar a la estrategia de marketing experiencial. Es decir, qué «Módulos Experienciales» se van a seleccionar para generar una experiencia. Después, sería necesario tomar decisiones acerca de la ejecución de cada MEE. Para facilitar esta toma de decisiones Schmitt ha diseñado una Matriz Experiencial de gran utilidad para planificar cualquier estrategia de marketing experiencial, que debe ser adaptada dependiendo de las necesidades y objetivos de planificación de cada empresa.

En este artículo se propone adaptar la Matriz Experiencial de Schmitt al diseño y la gestión de la experiencia de marca de un ECM.

Fig 1: Matriz Experiencial de un ECM

(Elaboración propia a partir de Matriz Experiencial de Schitt,2006)

\section{EX PROS}

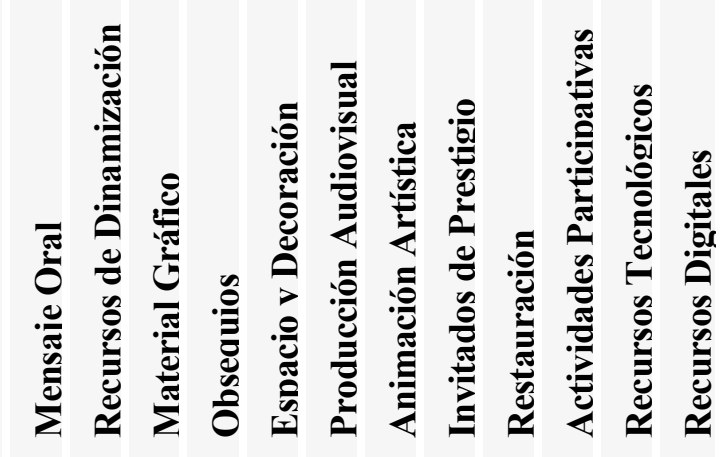

\section{SENSACIONES \\ M SENTIMIENTOS \\ E PENSAMIENTOS \\ E RELACIONES ACTUACIONES}

Esta matriz facilita el proceso de selección de los «Módulos Experienciales» que van a formar parte de la experiencia del evento. Y que van a ayudar a definir cómo será la experiencia que se quiere generar en el ECM. Esta experiencia podrá estar basada en un único «Modulo Experiencial», podrá ser un «Híbrido Experiencial» o podrá ser una 
«Experiencia Holística». ${ }^{27} \mathrm{Si}$ es posible se deberían crear experiencias que posean al mismo tiempo cualidades de sensaciones, sentimientos, pensamientos, actuaciones y relaciones.

Una vez que se ha tomado la decisión de qué «Módulos Experienciales» se van a utilizar para diseñar la experiencia del evento, habrá que tomar decisiones acerca de cada uno de ellos.

Para el «Módulo de Sensaciones», se debería decidir qué sensaciones debería experimentar el público objetivo. Es decir, si perseguir un enfoque estético o un enfoque de entretenimiento, y a que sentidos estimular.

Para el «Módulo de Sentimientos», habría que decidir qué estado de ánimo o que sentimiento concreto se pretende provocar.

Para el «Módulo de pensamientos», se tomarían decisiones relativas a qué pensará o imaginará, y qué aprenderá el público asistente al ECM.

Para el «Módulo de actuaciones, habrá que decidir qué tipo de experiencias físicas o interactuaciones con la marca o productos, se podrían compartir.

Para el «Módulo de relaciones», se debe decidir qué relaciones tendrán los asistentes. Estas decisiones estarán relacionadas con sus grupos de referencia, y cómo llevarles a identificarse con ellos, o cómo ayudarles a crear «comunidades de marca».

A partir de la definición del «híbrido experiencial» o la «experiencia holística» que debería tener el público asistente al evento, se debe decidir qué recursos se van a utilizar para crear y gestionar estas experiencias. Los «ExPros» son los componentes tácticos que tiene a su disposición el profesional de marketing para crear experiencias. En el caso de los ECM, los Expros son los «Recursos para el diseño de la experiencia de un $\mathrm{ECM} »^{28}$. Que son los recursos con los que los profesionales pueden contar para diseñar la experiencia.

En esta fase, el diseñador tiene que poner toda su capacidad creativa al servicio de la experiencia, y definir cómo debe ser cada uno de los Expros para que se consiga generar cada uno de los MEEs. La Matriz Experiencial de Schmitt puede servir para orientar la creatividad, y para ayudar a tomar decisiones sobre qué ExPros utilizar, y cómo utilizarlos para actuar sobre cada MEE definido.

Algunos autores, como Brown proponen considerar tres fases de la experiencia del evento $^{29}$. Para diseñar y gestionar la experiencia de un ECM resulta operativa esta división de la experiencia en tres fases:

27 Tal y como propone Schmitt: "Lo ideal sería que los profesionales del marketing se esforzaran estratégicamente para crear experiencias integradas holísticamente que posean, al mismo tiempo, cualidades de sensaciones, sentimientos, pensamientos, actuaciones y relaciones" (Schmitt, 2006:82-90).

28 Torrents propone una lista de recursos que pueden ser utilizados para diseñar y organizar un evento, que se ha actualizado y completado para enmarcarla dentro del contexto del diseño de experiencias. TORRENTS, R. (2005): Eventos de Empresa. El poder de la comunicación en vivo. Deusto, 112 83-88.

29 BROwn, S. (2001): «Torment your customer (they'll love it)». Harward Bussines Review, October, 
- Convocatoria. En este momento el público objetivo tiene que ser invitado a tomar parte. Por tanto, el ECM se tiene que ser diseñado para conseguir atraerle haciéndole ver la posibilidad de vivir una experiencia interesante, y que sólo se puede vivir una vez. Para ello se cuanta con medios tradicionales (invitaciones por correo o e-mail, o convocatorias en los medios de comunicación de masas), y con medios digitales (una web, un blog del evento, difusión en redes sociales, invitaciones a través de sms, etc.)

- Experiencia durante el Evento. Esta fase consiste en la experiencia del evento en sí misma. Es el momento en que el público objetivo (que respondió a la invitación) está emocionalmente enganchado con la marca. Para diseñar la experiencia durante el evento, se tienen que utilizar los recursos de forma creativa, a partir de un eje argumental, estableciendo un ritmo adecuado, intentando sorprender y ser memorable, manteniendo una coherencia entre los soportes, innovando en los formatos, y creando experiencias significativas.

- Difusión de la Experiencia. En esta etapa se tratará de sacar el máximo partido a la experiencia vivida por los participantes, y difundirla a través de otras herramientas de IMC. Es probable que los participantes sean un grupo relativamente pequeño, y que el evento pueda suponer un presupuesto elevado. Por tanto se trataría de expandir al máximo la experiencia a otros grupos de interés.

Se pueden utilizar herramientas de comunicación digitales, para que la experiencia perdure en la mente de los participantes, y se pueden diseñar campañas de marketing viral para difundir parte de la experiencia del evento.

De esta forma se puede utilizar la Matriz Experiencial para diseñar la experiencia de un ECM. Pero una vez vivida la experiencia se debería evaluar su resultado, ya que es la única forma de confirmar que la experiencia creada y compartida en el evento ha conseguido unos objetivos propuestos para un público objetivo determinado. Para ello, en la planificación del evento se habrán fijado unos objetivos concretos de la experiencia del evento, dentro de la estrategia integrada. Sólo si están claramente definidos los objetivos de la campaña de IMC, entonces se podrá delimitar qué papel le corresponde al evento dentro de la estrategia integrada.

Por tanto, si se realiza bien la planificación y el diseño de la experiencia de marca de un ECM, la relación del público con esa marca habrá mejorado para siempre, y su percepción de la marca ya no será igual que antes ${ }^{30}$.

La capacidad de los ECM para generar experiencias de marca, y su efectividad al integrarlos con otras herramientas de comunicación de marketing, están suponiendo un aumento en el uso de esta herramienta en muchas estrategias de IMC. El reto está en mantener esta situación, y continuar creando eventos innovadores y originales, que sigan teniendo valor para los participantes ${ }^{31}$.

\footnotetext{
30 Sснміт, B. H. (2003): Customer Experience Management, New York, John Wiley \& Sons.

31 Ibídim, 8.
} 


\subsection{Los eventos como conductores de la integración}

En la Universidad de Northwestern se está investigando sobre la posibilidad de desarrollar un modelo de planificación de las IMC en el que los ECM jueguen un papel fundamental como canalizadores de la integración. De hecho, según Fehrnstrom y $\mathrm{Rich}^{32}$, el marketing de eventos ha demostrado ser una herramienta bastante efectiva como conductor de la integración de las comunicaciones de marketing.

Los eventos pueden actuar como un elemento central de una campaña, y hacer de nexo con otras herramientas para optimizar la experiencia de marca. Para conseguir esto se requieren cambios fundamentales en la forma de trabajar de las organizaciones. Se tienen que sumar todas las capacidades de trabajo de las partes que intervienen en el proceso, y modificar como están estructurados los diferentes departamentos y agencias que intervienen en él.

Fehrnstrom y Rich realizan una propuesta de un modelo estratégico en el que los eventos trabajan como nexo para conducir hacia la integración de las comunicaciones de marketing. Para ello analizan el programa desarrollado por la empresa CISCO Systems, especializada en el desarrollo de programas, junto con George P. Johnson, especialista en marketing experiencial.

Después de años de debate profesional y académico, se ha llegado a la conclusión de que las IMC requieren algo más que un mero ajuste de planes tácticos que incluyan diferentes disciplinas. Las verdaderas IMC deben replantearse el proceso de planificación de las comunicaciones de marca, y construir la integración a partir de un planteamiento estratégico. Y, es ahí, donde los eventos pueden tener un papel fundamental.

En el entorno empresarial actual, las empresas ven a las IMC como una herramienta que permite desarrollar campañas de comunicación de marketing más eficientes, pero con un coste por impacto mayor. Son campañas que buscan relaciones con el consumidor a largo plazo. Y su objetivo es llegar hasta la fase de adquisición, a través de la afectividad y la conexión con la marca ${ }^{33}$.

Hablamos de un modelo integrado de comunicación de marketing que se centra en relaciones con el consumidor a largo plazo, y que no utiliza nunca las herramientas de forma individual, ya que pretende actuar tal y como los consumidores perciben un mensaje global de marca.

La realidad actual muestra que existen caminos impredecibles por los que los consumidores reciben la información, y que estos influyen en sus decisiones. Si estos caminos no están controlados, y si los recursos están fragmentados, entonces va a ser muy difícil combinar las correctas interacciones para la marca en las diferentes etapas del proceso de compra. A la inversa, si se controlan los mensajes sobre la marca, en el momento adecuado, y seleccionando los mejores medios, se podrá conseguir un cambio de actitud, y una duradera preferencia de marca. La integración puede ser un ca-

32 Fehrnstrom, M. y Rich, D. (2009): «Using events to drive an Integrated Marketing Model». Northwestern University, Journal of Integrated Marketing Communications

33 Ibídim, 30. 
mino muy rentable para conseguir «una experiencia real transformadora» que permita alcanzar unos objetivos previstos ${ }^{34}$.

Por tanto, para que las promesas integradoras de las comunicaciones de marketing puedan ser una realidad sería necesario racionalizar el proceso. El equipo de CISCO System ha desarrollado un marco de referencia para planificar programas integrados eficientes de marketing experiencial. Estos programas integrados parten de un proceso llamado «Porfolio Planning»»» que podría equivaler al servicio de planificación de medios, pero se realiza dentro de un marco experiencial. Es un sistema de planificación que se basará en la identificación de cómo experimentan durante el proceso de decisión los públicos objetivos. Dentro de esta perspectiva se desarrolla el «Mapa Estratégico de Experiencia» (SEM), que supone uno de los elementos clave del programa estratégico. SEM conceptualiza de forma detallada las interacciones con en público objetivo y sus sub-segmentos.

Este planteamiento, por tanto, necesita profundizar en el conocimiento de los resortes emocionales y racionales del público objetivo, para poder conseguir los objetivos concretos.

Fig 2: SEM: Mapa Conceptual Cisco Systems (Fehrnstrom y Rich, 2009)

Advertising
Analyst Rela-
tions
Public Rela-
tions
Direct Marke-
ting

Events

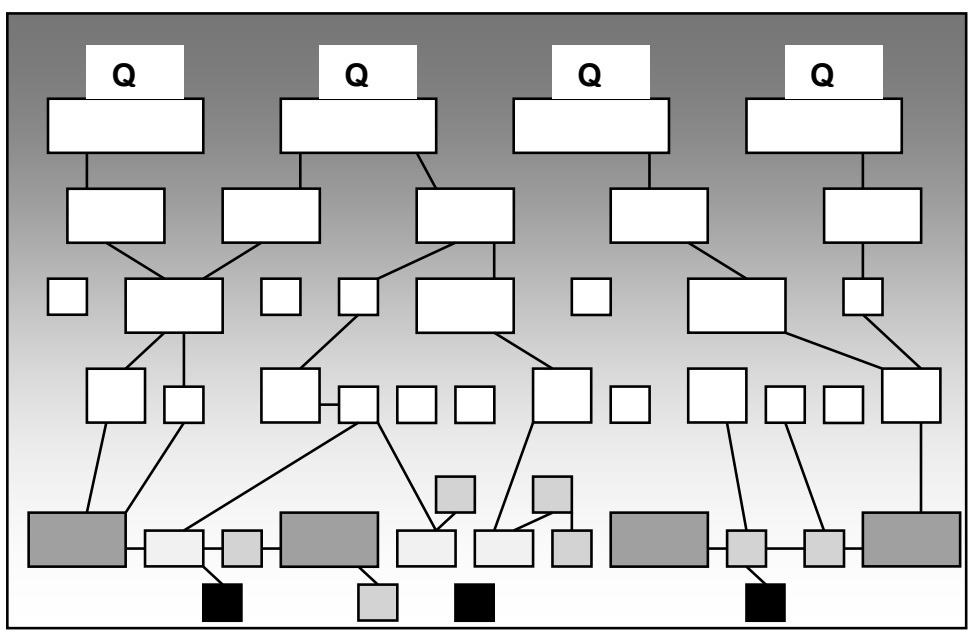

Este «Mapa Conceptual» explica como las experiencias diseñadas crean reacciones en el público objetivo. Y busca el número y el tipo de interacciones de marca necesarias para conseguir el objetivo. Para ello utiliza varias herramientas que van a ser necesarias para llevar al publico en una dirección concreta.

34 GiLmore, J.H. \& Pine, J. (2007): Authenticity: What Consumers Really Want. Harvard Business School Press. 
Por tanto «SEM» $\mathrm{y}$ «Porfolio Planning» son las herramientas que ayudan al equipo de CISCO a diseñar verdaderas campañas integradas, centradas en la experiencia del público, que pueden incluir de forma detallada cada una de las herramientas de IMC.

Es decir, el resultado es un modelo de planificación de las comunicaciones de marketing totalmente integrado, que está activado por múltiples herramientas de comunicación de marketing, en la secuencia apropiada y con el grado exacto.

El planteamiento de CISCO es muy innovador ya que reúne, desde las primeras etapas, a los diferentes equipos multidisciplinares. Estas reuniones consiguen sumar la experiencia y la capacidad de los diferentes equipos: especialistas en publicidad, en relaciones públicas, en comunicaciones online, en organización de eventos, etc. Involucra a estos equipos de forma conjunta y les obliga a buscar consensos. Así, la planificación resultante es una visión unificada de las comunicaciones de marketing, y un reconocimiento de la contribución de cada herramienta en el plan de IMC.

$\mathrm{El}$ «Modelo SEM» parte del hecho de que los eventos requieren de otras herramientas de comunicación de marketing. Esta es una de las claves del uso de los eventos como un punto central de la planificación de las IMC. Considera que los ECM no pueden actuar solos ya que necesitan de otras herramientas de IMC, en parcelas como: la generación de audiencias, la pre-cualificación, las comunicaciones post-evento; y las investigaciones post-evento.

Una de las características de los ECM es que tienen lugar en un momento y en un tiempo específico. Por tanto, pueden ser de alguna forma un punto de encuentro de un sector de actividad o de «una comunidad-estilo de vida».

Los autores ${ }^{35}$ utilizan como ejemplo para la aplicación del Modelo el lanzamiento de un nuevo producto en una feria de tecnología. En ella, una comunidad de consumidores, medios e influenciadores van a estar juntos en el mismo sitio y al mismo tiempo. Consideran la asistencia a la feria como una herramienta muy útil para construir una campaña de IMC a su alrededor. Y este «lanzamiento suave», puede resultar mucho más eficaz que utilizar un lanzamiento masivo mucho más caro. Y puede ser una buena referencia como nexo de la campaña.

Este ECM, por tanto, va a ser una herramienta centralizadora y unificadora, perfecta para desarrollar el trabajo de múltiples especialidades de comunicación dirigidas a un objetivo común. Se unen los equipos multidisciplinares y aportan cada uno su experiencia. En conclusión, se unifican todas las herramientas de comunicación de marketing a partir de un evento de comunicación de marketing, en un tiempo específico.

A partir de este análisis del Modelo SEM, y de su puesta en práctica para una campaña concreta, Fehrnstrom y Rich concluyen que el marketing experiencial debe ser el nexo de todas las herramientas de IMC. Por tanto, la relación de interdependencia entre la estrategia de marketing experiencial y las herramientas de IMC hacen del ECM un medio natural para hacer de nexo entre todas las herramientas. En el contexto actual

35 Ibídim, 32 
de las IMC los eventos están pasando de ser una pura táctica, a servir como punto clave estratégico desde el que enfocar una campaña de comunicación integrada.

El Modelo de Planificación de CISCO plantea la posibilidad de que los profesionales de los ECM y los expertos en IMC, crucen sus disciplinas para pensar estratégicamente en el papel de los eventos en el mix de las comunicaciones de marca ${ }^{36}$.

\section{Los objetivos de la experiencia de un ECM}

Para poder delimitar el papel que le corresponde al evento dentro de la estrategia integrada, los objetivos deben estar claramente definidos. Tanto los objetivos de IMC, como los objetivos de la experiencia del evento.

Por lo tanto, es necesario definir qué efectos se pretenden a partir de la experiencia del público objetivo en un ECM.

Sí el evento se dirige a diferentes públicos objetivos (prescriptores y clientes actuales), puede ser necesario definir unos objetivos diferentes para cada público. Para delimitar los objetivos de la experiencia del evento, el organizador tendrá que planificar los efectos que espera producir en el público objetivo a partir de la experiencia.

Los ECM siempre suponen una inversión que sólo se podrá justificar en función de unos objetivos y unos resultados, por lo que será necesario definir los posibles objetivos para que la utilidad de esta herramienta pueda ser evaluada de forma creíble.

Las mediciones actuales de atención, cobertura mediática y satisfacción no son suficientes para evaluar un ECM. Habría que buscar unos parámetros de medida que sean capaces de evaluar los verdaderos efectos de los eventos. Para ello, va a ser necesario contextualizar la evaluación dentro del marketing experiencial, y plantear la evaluación a partir de objetivos enmarcados dentro de la experiencia de marca del evento ${ }^{37}$.

Para realizar este artículo se han revisado los planteamientos de varios autores respecto a los objetivos experienciales de los ECM.

Masterman y $\mathrm{Wood}^{38}$, consideran que la experiencia que se crea en un ECM puede tener por objetivo desarrollar relaciones, asociar la marca a buenas causas que interesen al público objetivo, y/o crear, cambiar y reforzar la imagen de la marca, productos o de la organización.

Lenderman $^{39}$ define como un objetivo específico de los ECM el «alcanzar un grado de intimidad » entre la marca y el público objetivo. Esto sólo se puede conseguir a partir de una experiencia de marca, que supone una interacción personal e intima entre el público objetivo y la empresa. Considera que otro de los objetivos experienciales de un evento es crear «rumor» y amplificar la experiencia del evento. A los eventos, tanto

\footnotetext{
36 Ibídim, 32.

37 Ibídim, 14.

38 Ibídim, 14

39 Lenderman, M. (2008): Marketing Experiencial. La Revolución de las Marcas, Madrid, Editorial ESIC, 80-85.
} 
los «influenciadores» como los «conectores», son invitados en exclusiva, y asisten de manera voluntaria. Saben que van a tener información de primera mano y esto les anima a generar el «boca a boca». Pero la experiencia del evento les debe inspirar y motivar para que hablen de la marca.

La clave del «Buzz marketing» está en llegar a muchos conectores estratégicos, y para ello hay que considerar a las personas como parte de una red. Rosen considera que el objetivo es acceder directamente a los núcleos mas estratégicos de la red ${ }^{40}$. Es aquí donde los ECMs pueden tener un papel importante. Si un evento es capaz de acceder a este «núcleo de la red» y generar interactuaciones y experiencias positivas, es posible que el rumor se extienda con gran rapidez.

Para Lenderman esta interacción con el público objetivo puede ir aun más allá. El evento puede tener por objetivo crear «comunidades de marca», o reforzar las relaciones entre estas comunidades. Puede hacer que los «prossumers» se conozcan entre sí, comparta momentos y experiencias en torno a una marca.

Además, los eventos son una herramienta muy útil para establecer «conversaciones» con los clientes. Permite comunicarse con ellos a través de experiencias y obtener retroalimentación ${ }^{41}$. Por tanto, otro objetivo de la experiencia del evento puede ser obtener respuestas del consumidor. Esto se puede conseguir, ofreciéndoles experimentar libremente con las marcas y con los productos, y analizando posteriormente sus sensaciones y emociones. Cuando los «embajadores de marca» conversan con los asistentes a un ECM, se produce un diálogo de un valor incalculable. Tanto el público, como el representante de la marca pueden aprender uno de la experiencia del otro. Es posible, que los «embajadores de marca» interactúen con los clientes y les influyan sobre sus percepciones y sobre su comportamiento.

Como conclusión, podemos decir que cuando se diseña una experiencia de un evento se pretende conseguir, en primer lugar, una experiencia satisfactoria con la marca, productos, u organización. Y, a partir de ella, alcanzar unos objetivos que puedan ser evaluados.

Los «Objetivos de la experiencia de un ECM» se pueden clasificar en tres categorías:

- Generar Cobertura mediática o generar «rumor».

- Crear o fortalecer la relación experiencial con la marca, para crear relaciones sólidas y duraderas con ella.

- Motivar a una determinada conducta: a «evangelizar» sobre la marca, a realizar determinados esfuerzos de venta, a realizar mejor una labor profesional, a relacionarse entre sí y con las marcas creando «comunidades de marca», etc.

Estos objetivos, se deben plantear teniendo en cuenta que su consecución pueda ser evaluada. Para lo que es necesario que sean específicos, medibles, aceptados, realistas, temporalizados y definidos en un contexto integrado y experiencial.

40 Rosen, E. (2009): The Anatomy of Buzz Revisited: Real-Life Lessons in Word-Of-Mouth Marketing. Broadway Books.

41 Ibídim, 13. 


\section{La evaluación de la experiencia de un ECM}

Masterman y Wood ${ }^{42}$ han investigado sobre la evaluación de los ECM, y para ello han analizado los puntos de vista de algunos expertos en comunicaciones de marketing con una gran experiencia en organizar y evaluar ECM.

Estos profesionales dan una gran importancia a la naturaleza emocional de los eventos, y consideran que la experiencia creada en el ECM es fundamental para su éxito. Por tanto, será necesario evaluar los ECM desde una perspectiva experiencial, y para ello hay que localizar las conexiones emocionales producidas a partir de la experiencia del evento.

En lo que se refiere a los tipos de evaluación y los métodos para evaluar, los profesionales consideran que la profundidad y amplitud de las evaluaciones varía mucho dependiendo de las necesidades de los clientes y de su disposición a pagar. Los métodos que hoy por hoy se están utilizando para evaluar los ECM pueden ser tanto cualitativos como cuantitativos. Admiten que la mayoría de las evaluaciones se centran en el evento en sí mismo, más que en sus efectos. Y reconocen que sería fundamental realizar una evaluación más profunda, en la que se evalúen los efectos de los ECM.

Por tanto, la falta de profundidad y amplitud de las evaluaciones es un problema que afecta al sector de forma generalizada. Y los entrevistados en este estudio consideraron sería necesario que existieran una serie de métodos de investigación eficaces que se pudieran adaptar a la evaluación de los ECM, permitiendo desarrollarlos y crecer con ellos. Además, sería necesario un cambio de planteamiento y un compromiso, dentro del sector, con la evaluación de los eventos.

Por tanto, la investigación de Masterman y Wood deja patente la necesidad de investigar para encontrar métodos para evaluar los ECM desde el valor de la experiencia.

A lo largo de los últimos años, los psicólogos y los investigadores de marketing han desarrollado metodologías para evaluar las experiencias de los públicos. Estos métodos tratan de medir sensaciones, sentimientos, pensamientos, actuaciones y relaciones. Estas técnicas se están utilizando para evaluar los resultados de la estrategia global de marketing experiencial de una organización, y para evaluar algunos aspectos experienciales de varios ExPros.

Schmitt. Brakus y Zarantello ${ }^{43}$ han creado una breve escala para medir la experiencia. Ha sido probada en cuanto a fiabilidad y validez, y podría servir para evaluar en que grado un ECM es capaz de influir sobre la «relación experiencial» del público con una marca.

Es difícil plantar unas normas sobre la evaluación de los ECM, debido a la gran variedad de tipos diferentes de ECMs. Pero se podrían establecer unas pautas para su evaluación, centrándose en la experiencia de los consumidores en el evento, teniendo

42 Ibídim, 14.

43 En Schmitt et all 2008, se resume el trabajo de investigación realizado por Schmitt, Brakus y Zarantello en la Universidad de Columbia, en el que se desarrolla la "Escala de la Experiencia" (Exscala) que permite evaluar la relación experiencial de los públicos con las marcas. 
en cuenta que los métodos sólo podrían ser aplicables si previamente se han definido unos objetivos concretos de la experiencia del evento. La efectividad global del evento debe requerir un tipo de evaluación diferente según los objetivos planteados.

La evaluación del ECM debe centrarse en investigar «cómo ha sido la experiencia de los consumidores en el evento». Y, más importante aún, en «las respuestas a largo plazo a esta experiencia».

Aunque sería casi imposible desarrollar una metodología de evaluación aplicable a todos los ECM, sí se podría plantear un modelo metodológico aplicable en cada caso, a partir de una definición concreta de los objetivos de la experiencia del evento, y de los objetivos de IMC. De cualquier forma, siempre va a ser mejor intentar evaluar la experiencia del ECM con los métodos disponibles, que dejar de evaluarla ${ }^{44}$.

A partir del análisis realizado, proponemos un posible sistema de evaluación para los ECM, aplicable de forma individual, en cada caso, teniendo en cuenta diferentes factores de cada ECM, como son: el tipo de ECM, las características y los objetivos del público objetivo, otras herramientas de IMC, los objetivos IMC y los objetivos experienciales, así como los recursos disponibles para la evaluación (tiempo y dinero). $\mathrm{Y}$ otros factores secundarios, como el tiempo de contacto con los asistentes, las posibilidades de contactar con ellos después del evento, y el tipo de relación que tiene la empresa con los asistentes.

El sistema de evaluación que se plantea tiene en cuenta las categorías de objetivos definidas anteriormente, así como la evaluación de los resultados de cada una de las fases de la experiencia de un ECM.

- Evaluación de la Convocatoria. El objetivo de la convocatoria a la experiencia se debe establecer en términos de asistencia al evento. Para evaluar su eficacia se debe medir cuántos y qué características tienen las personas que han respondido a ella.

En la mayoría de los eventos no es tan importante el número como el perfil de los asistentes. La convocatoria es un éxito si consigue atraer al número de asistentes previsto, y si el perfil de éstos coincide con el perfil del público objetivo definido.

En esta etapa los métodos de evaluación suelen ser métodos cuantitativos sencillos que permitan controlar la asistencia al evento. Se hace un poco más complicado en los ECM en que la convocatoria es abierta y el contacto con el asistente es escaso. Pero, incluso en estos casos, hay que buscar fórmulas para identificar a las personas que van a tener experiencia con la marca durante el evento.

- Evaluación de la Experiencia durante el ECM. Los objetivos de la experiencia durante el evento pueden ser muy diferentes en cada caso.

Pero van a ser considerados dentro de dos categorías: «reforzar una relación experiencial con la marca», o «motivar a realizar una determinada conducta». Dentro de estas dos categorías se puede plantear uno o más objetivos concretos temporalizados y medibles.

44 Ibídim, 14. 
Para concluir en qué medida los objetivos definidos se han conseguido, se puede utilizar cualquier método de evaluación que permita medir un objetivo determinado. Por ejemplo, se puede entrevistar «a posteriori» a los asistentes para saber si están interesados en concertar una entrevista con el equipo de ventas. O se podría utilizar el método propuesto por Reichheld ${ }^{45}$ y preguntar a los asistentes por su intención de prescripción. $\mathrm{O}$, para evaluar la relación experiencial en un evento de larga duración, se podía pedir antes de la experiencia valorar la «escala de la experiencia», y una vez finalizada la experiencia del evento ver si esa valoración ha sido mejorada. Incluso se podría seleccionar sólo una muestra del público asistente para valorar la escala. «»

El organizador del evento tiene que conocer todos los posibles métodos para evaluar, y decidir, en cada caso, que método aplicar.

- Evaluación de la Difusión. Los objetivos de la difusión se plantean en términos de apariciones en los medios de comunicación tradicionales, o en presencia en Internet. Se pueden prever unas apariciones determinadas en los medios de comunicación de masas, así como una presencia concreta en determinadas redes sociales, blogs y sitios web. Para medir la difusión mediática, se puede realizar un «clipping» de medios, para controlar en qué medios y cómo, aparece el ECM. Y para evaluar la difusión en Internet, se puede realizar un «rastreo" o «trucking» que controle las apariciones del evento en la red.

En definitiva, sólo una evaluación rigurosa de los resultados, tanto interna como externa, hace posible medir la efectividad de la experiencia del evento. Y, por tanto, hace justificable la organización de un ECM, dentro de una estrategia de IMC.

El futuro de los ECM va a depender, en gran medida, de la capacidad para evaluar los resultados de esta herramienta. Y las posibilidades de evaluación - esta es la propuesta de este artículo- tienen que concebirse dentro de contextos experienciales e integrados.

\section{Conclusiones}

El valor de los eventos, en la estrategia de IMC, reside en su capacidad para generar y compartir experiencias de marca significativas con los diferentes públicos de las organizaciones que les permitan alcanzar unos objetivos concretos. Estos pueden ser: mejorar o reforzar las relaciones con la marca, empresa y productos; conseguir un refuerzo o cambio de actitud; o motivar a una conducta determinada. De cualquier forma, para conseguir experiencias completas y ampliadas los ECM necesitan integrarse con otras herramientas en la generación de audiencias, en la pre-cualificación de los asistentes, en las difusión de la experiencia post-evento, y en las investigaciones post-evento.

Para generar estas experiencias de marca significativas los profesionales tienen que conocer en profundidad a los asistentes a un ECM, y sus objetivos experienciales. Así mismo deben controlar todos los Expos que les van a permitir diseñar experiencias

45 ReIChHeld, F (2006): The Ultimate Question. Driving Good Profits and true growth, Hardvard, Hardvard Business School Press. 
holísticas a partir de los MEEs de sensaciones, de sentimientos, de pensamientos, de actuaciones y de relaciones.

Hoy por hoy, los responsables de comunicación de las organizaciones son conscientes del valor experiencial de los eventos y de su capacidad de integrarse en la estrategia de IMC, pero no tienen claro su papel en la misma. Para avanzar en ello, es necesario que se empiecen a planificar las campañas a partir de objetivos experienciales e integrados, y que se utilicen métodos capaces de evaluar la consecución de estos objetivos.

Las investigaciones que se están realizando en ámbitos académicos anglosajones ${ }^{46}$ pueden servir como punto de partida para seguir investigando sobre estos métodos y sobre cómo planificar campañas de IMC experienciales en las que los ECM pueden tener un papel estratégico fundamental.

En definitiva queremos concluir genéricamente, al mismo tiempo que apuntamos dos posibles líneas de investigación con altas implicaciones de tipo antropológico, que:

- La experiencia es integradora porque es humana y el ser humano es integrador. De la misma forma, la integración de las herramientas cada día tiene más sentido en contextos experienciales en los que se busca crear y compartir experiencias de marca con los públicos.

- La integración es en sí misma ya una experiencia: Al integrar -o percibir la integración- ponemos en funcionamiento nuestros sentimientos, sensaciones, pensamientos, relaciones y, finalmente, actuaciones. $Y$ esto de forma sinérgica con el mensaje. Y de forma privilegiada -como se ha tratado de exponer en el artículo- a partir de la organización de un evento.

\section{Referencias bibliográficas}

ACEBAL NeU, A. (2004): «Eventos: Un instrumento de comunicación al servicio de la empresa», Bilbao, Ediciones Deusto (e- Deusto Dossier).

Belch, G. Y M. BelCh. (2009): Advertising and Promotion: An Integrated Marketing Communications Perspective, 6th Ed. McGraw-Hill, San Diego State University.

BERRIDGE, G. (2007): Events Design and Experience, Oxford, Elsevier Buterworf-Heinemann (Events Management Series)

BROwN, S. (2001): «Torment your customer (they'll love it)», Harward Bussines Review, October, 83-88.

CAYWOOD, C.L. (1997 ): The Handbook of Strategic Public Relations \& Integrated Communications. McGraw-Hill Professional.

Clow, K. Y BAACK, D. (2005): Integrated Advertising and Promotion and Marketing Communications, 2nd ed. Upper Saddle River, New Jersey, Prentice Hall.

FEHRNSTROM, M. Y RICH, D. (2009): «Using events to drive an Integrated Marketing Model». Northwestern University, Journal of Integrated Marketing Communications.

Grupo Eventoplus (2010): «El estudio del Mercado de Eventos 2010». Disponible en www.eventoplus.com

46 Masterman y WoOd, 2008; Schmitt et all, 2008; y FeShTrom y Rich, 2009 
GilmoRe, J.H. \& Pine, J. (2007): Authenticity: What Consumers Really Want, Harvard Business School Press.

GuTIÉRREZ ZÚMEL, C. (2004): El evento: un plus en comunicación, Bilbao, Ediciones Deusto Planeta de Agostini Profesional y Formación.

Holm, O.. (2006): «Integrated marketing communication: from tactics to strategy». Corporate Communications. International Journal, vol. 11, 1, 23-33.

Kitchen, J. y De Pelsmacker, P (2004): Integrated Marketing Communications. A primer. New York, Routledge.

LENDERMAN, M. (2008): Marketing Experiencial. La Revolución de las Marcas, Madrid, Editorial ESIC.

MASTERMAN, G. \& WoOD, E. (2006): Innovative Marketing Communications: Strategies for the Events Industry, Oxford, Elsevier Buterworf-Heinemann.

MASTERMAN, G. \& WoOD, E. (2008): «Event Marketing: Measuring an experience». Venice: 7 th International Marketing Trends Congress - Jan 17th/19 .

Molero, V. Y Moreno, F. (2008): «Marketing de Vanguardia», en Manual de Marketing, Madrid, Editorial ESIC.

PickTON, D. \& BRODERICK, A. (2005): Integrated Marketing Communications, London, Prentice Hall. 2nd Edition.

ReICHHELD, F (2006): The Ultimate Question. Driving Good Profits and true growth, Harvard, Hardvard Business School Press.

RodríGUEZ ARDUA, I. (2007): Estrategias y Técnicas de Comunicación. Una visión integrada en el Marketing, Madrid, Editorial OUC.

Rosen, E. (2009): The Anatomy of Buzz Revisited: Real-Life Lessons in Word-Of-Mouth Marketing. Broadway Books.

Schmiтt, B. H. (2003): Customer Experience Management, New York, John Wiley \& Sons.

SCHMITT, B. H. (2006): Experiential marketing : cómo conseguir que los clientes identifiquen en su marca sensaciones, sentimientos... relaciones. Bilbao, Ediciones Deusto, 2006.

Schmitt, B. H.; Rogers, D. L. Y VROTSOS, K. (2003): There's No Business That's Not Show Business: Marketing in an Experience Culture, Publisher, FT PressPub.

SChMitT , B. H; BRAKUS, J. \& ZARANTONELlO, L. (2008): «Brand Experience: What is It? How do We Measure It? And Does It Affect Loyalty?», Journal of Marketing, American Marketing Asociation.

Schultz, E. \& Schultz, H. (2004): IMC, the next generation: five steps for delivering value and measuring financial returns using marketing communication, New York, McGraw-Hill.

TORRENTS, R. (2005): Eventos de Empresa. El poder de la comunicación en vivo, Bilbao, Ediciones Deusto.

VictoriA, J.S. et al (2005): Reestructuras del Sistema publicitario, Barcelona, Ariel

WOHLFEIL, M. Y WHELAN, S. (2005): «Event Marketing: When Brands Become 'Real Lived' Experiences», Irish Academy of Management Conference, Galway-Mayo Institute of Technology. 